\title{
Quantitative Analysis of Simulated Erosion for Different Soils
}

\author{
Zhongxian Chen \\ chenz5@cs.rpi.edu \\ Jared Gross \\ grossj@rpi.edu
}

\author{
Christopher Stuetzle \\ stuetc@cs.rpi.edu \\ W. Randolph Franklin \\ franklin@cs.rpi.edu
}

\author{
Barbara Cutler \\ cutler@cs.rpi.edu
}

Thomas Zimmie zimmit@rpi.edu

\section{Rensselaer Polytechnic Institute Troy, NY}

\begin{abstract}
Levee overtopping can lead to failure and cause catastrophic damage, as was the case during Hurricane Katrina. We present a computer simulation of erosion to study the development of the rills and gullies that form along an earthen embankment during overtopping. We have coupled 3D Smoothed Particle Hydrodynamics with an erodibility model to produce our simulation. Through comparison between simulations and between simulation and analogous laboratory experiments, we provide quantitative and qualitative results, evaluating the accuracy of our simulation.
\end{abstract}

\section{Categories and Subject Descriptors}

I.3.5 [Computer Graphics]: Computational Geometry and Object Modeling - physically based modeling;

I.6.4 [Simulation and Modeling]: Model Validation and Analysis and Simulation Output Analysis

\section{Keywords}

hydraulic erosion simulation, physical modeling

\section{INTRODUCTION}

During Hurricane Katrina in 2006, the overtopping, seepage, and eventual failure of earthen levees protecting New Orleans, LA, caused vast devastation to the city and its surrounding areas. A better understanding of the evolution of rills and gullies, which form during levee overtopping, is necessary to enable the design of structures that can more effectively withstand storm conditions.

Erosion Literature During storm conditions, a levee overtops the moment the level of water has reached and exceeds the crest (highest part of the levee) and flows over the downslope side. Hanson et al. [7] presented a four-stage erosion process that occurs during overtopping of an embankment. Wang et al. $[12,13]$ presented two dimensional mathematical models for the erosion of an embankment. Briaud

Permission to make digital or hard copies of all or part of this work for personal or classroom use is granted without fee provided that copies are not made or distributed for profit or commercial advantage and that copies bear this notice and the full citation on the first page. To copy otherwise, to republish, to post on servers or to redistribute to lists, requires prior specific permission and/or a fee.

ACM SIGSPATIAL GIS 2010 San Jose, California USA

Copyright 20XX ACM X-XXXXX-XX-X/XX/XX ...\$10.00.

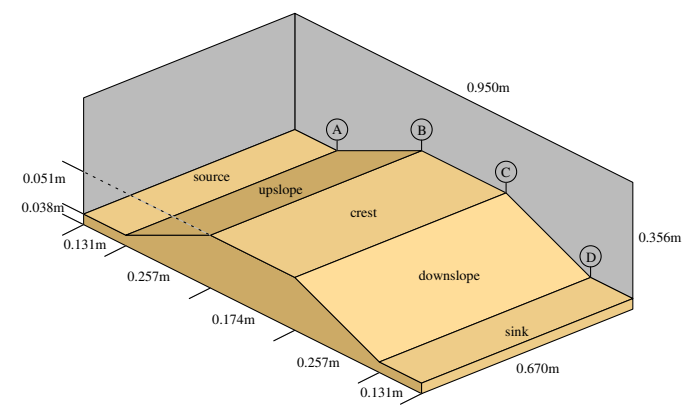

Figure 1: Diagram of the geometric dimensions of the levee for our computer simulations and physical experiments. Note the labels A, B, C, and D along the profile of the levee.

et al. [1] expanded the traditional definition of a soil's "erodibility" to account for water velocity that varies throughout the flow field, instead defining erodibility as a function of shear stress over the surface of the soil. This model is applicable to small-scale erosion simulations, and is the erosion model implemented in our simulation.

One important metric for the effectiveness of a levee is the average time to breach during storm conditions. Fread, from the National Weather Service, defined time to breach as the duration of time between the initial formation of a rill and the time at which the rill has reached the upslope of the levee, forming a clear channel along with breaching water runs [4]. We base our quantitative analysis of computer simulations and laboratory experiments on this accepted observational definition.

Computer Modeling of Hydraulic Erosion Fluid flow and hydraulic erosion simulations have been developed for computer graphics, though the primary goal has been to create physically plausible terrains mimicking features formed through erosion processes. Fluid simulation techniques can be divided into two categories. In the first category are gridbased Eulerian techniques, as used by Foster and Metaxas with the Marker-And-Cell method to solve 3-D Navier-Stokes equations [3]. An alternative to Eulerian methods, the high resolution particle-based Lagrangian methods based on Smooth Particle Hydrodynamics (SPH) [5, 9], is becoming more popular. Kristof et al. [8] was the first to present an erosion simulation using SPH. The soil, water, and soil-water boundary were all represented by particles, the soil and water particles have mass and velocity while the boundary particles are designed solely for the two phases to interact. This 


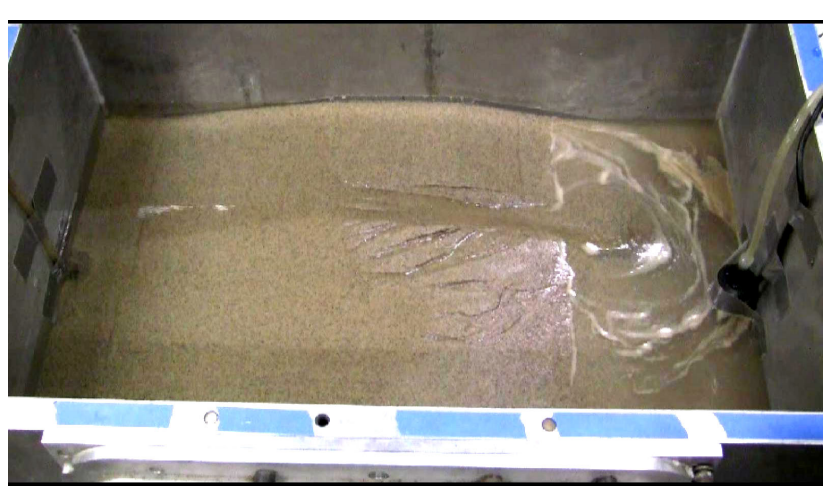

Figure 2: Physical experiment using pure sand.

method is most similar to our simulation method. To represent terrain, we use a Segmented Height Field (SHF) [10].

Evaluation and Validation One of the most important goals of our work is to evaluate the accuracy of our computer simulations through comparisons with experimental data, collected from laboratory trials. To our knowledge, validation of the accuracy of the detailed computer simulations of erosion, especially those for computer graphics use, has received little attention. One notable exception is the SODA project [11], in which a patch of soil was pelted with rain both in a laboratory and in a cellular automata computer simulation, and the results are visually compared.

\section{EXPERIMENTAL METHODOLOGY}

To study the formation and propagation of rills and gullies in the surface of an earthen embankment and enable validation of our computer simulations, we conducted a series of physical laboratory experiments, which are described in our earlier work [6], shown in Figures $1 \& 2$. We performed analogous computer simulations [2] for five different sets of erosion parameters that span the estimates of the soil parameters in our physical laboratory experiments (Figure 3). We performed two trials for each simulation as an initial investigation of the variance of these simulations and average their results.

Table 1 presents several interesting quantitative measurements for each simulation. We calculate the maximum vertical erosion depth and the total volume of eroded soil for each trial after ten minutes of simulation (from the point of initial overtopping). The last three columns of the table present the elapsed time for three specific milestones that indicate breach of the levee.

To characterize and evaluate the physical accuracy of our erosion simulation, we provide results for our computer simulation trials with different soil parameters and confirm that the system behavior changes as expected with regard to rill and gully formation, maximum erosion depth, total erosion volume, and two objective and quantitative time to breach metrics. Furthermore, we compare the results from our computer simulations to our laboratory laboratory erosion experiments.

\section{ANALYSIS AND DISCUSSION}

Comparison of Computer Simulations We analyze the results of our computer simulations of erosion by visualizing and comparing the erosion through different quanti-

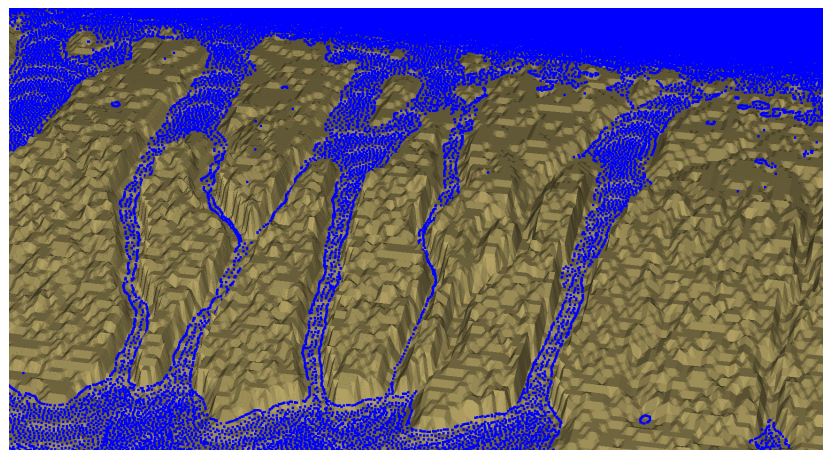

Figure 3: A computer simulation result showing deep channels in a soil with medium erodibility.

tative metrics, and draw conclusions on the validity of our erosion model and simulation results. As shown in Table 1, the values of maximum vertical erosion depth and total erosion volume at 10 minutes after the initial overtopping generally increase with increased erodibility and also, logically, the time to breach (for each of the three metrics) is shorter for soils with higher erodibility.

We perform a detailed analysis of several easy-to-monitor geometric and simulation properties. In Figure 4, we plot the values of maximum vertical erosion depth in different zones of the levee with respect to time. Each of the five plots presents results for a specific set of soil parameters. As can be observed from the plots, the crest of the levee is the most vulnerable to erosion when compared to the upslope and downslope and, as expected, the erosion is most aggressive on the downslope and the crest. Furthermore, the erosion depth for highly erodible soils increases dramatically in the first five minutes and then levels off. In contrast, the erosion depth for less erodible soils proceeds at a more constant pace throughout the 10 minute simulation.

Time to Breach Metrics To identify the moment of breach, we follow the somewhat subjective definition of the Dam-Break Flood Forecasting Model [4] (Table 1, 6th column). In addition to this classic definition, we propose two quantitative metrics related to levee breach that can be calculated directly from the computer simulations, illustrated in Figure 5. In the left plot, we monitor the upslope face of the levee to determine when significant erosion occurs in this zone, as this will indicate the formation of a channel across the crest of the levee. We define the moment of breach as the moment when this upslope erosion exceeds a specific threshold (Table 1, 7th column). Next, we observe that levee breach is typically accompanied by a dramatic increase in the magnitude of the velocity of water particles crossing the crest of the levee. In our simulation, velocity of water flow can be approximated by the average velocity of individual water particles in the zone of the levee crest (between positions $\mathrm{B}$ and $\mathrm{C}$ in Figure 1). In the right plot of Figure 5, we observe that the velocity for simulations with larger erodibilities peaks earlier than for those with smaller erodibilities (Table 1, 8th column).

Visual Comparison of Erosion In Figure 6, we present a visual comparison of the development of the number, shape, branching pattern, and depth of the rills and gullies in different computer trials. Several interesting observations can be made from these images. First, as the erodibility of the soil increases, the gullies become deeper and wider. Early in 
sand-clay mixture

$\# 1: a=93, \tau_{c}=3.00$

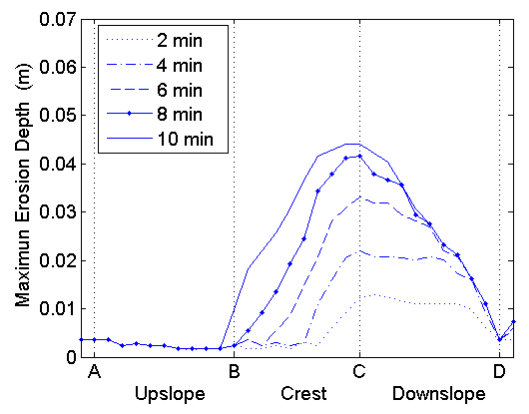

$\# 3: a=137, \tau_{c}=2.50$

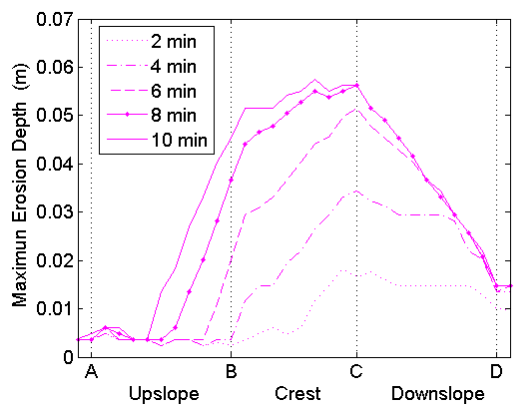

pure sand

$\# 5: a=187, \tau_{c}=2.00$

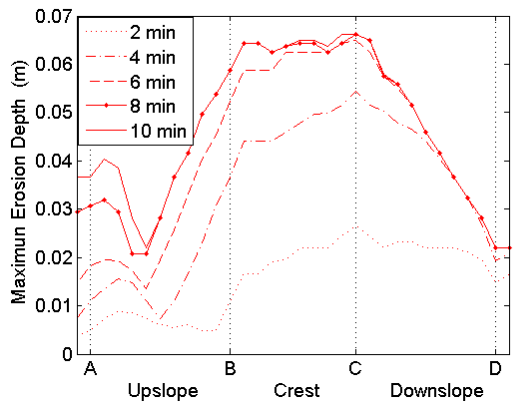

Figure 4: We plot the maximum erosion depth along the length of the levee for the different soil types. The less erodible soils display a smaller maximum depth of erosion and a smaller total volume of erosion (not shown). Note that in all cases the erosion begins on the downslope of the levee, progresses across the crest of the levee, and finally erodes the upslope to breach the levee.

the trial with highly erodible soil we can also see numerous small channels, but as the erosion progresses fewer, deeper primary channels emerge, allowing the secondary channels to dry up. If we continued the trials beyond 10 minutes, this pattern may well follow for the less erodible soils as well. Because the water's velocity should be greatest at the base of the downslope (point D), yielding higher shear stress and maximum erosion, we expect to first observe erosion at the base of the downslope and then watch it progress up to the crest of the levee (point C). However, in our computer simulations the erosion on the downslope was uniform from crest to base and ultimately the greatest depth of vertical erosion occurs along the crest and the top part of the downslope. These observations may be due to the overall scale and proportions if the geometry. For simulations of full-scale levees (for which we will create analogous simulations with small-scale models using our geotechnical centrifuge [14]) we expect to see increased velocities and more significant initial erosion at the base of the downslope, and possibly more varied channel formation.

Comparison of Computer Simulations and Physical Experiments Finally, we compare our computer simulation trials with the laboratory experiment shown in Figure 2. During the erosion of the physical model, the time to breach was 6:25 for fine-grain sand with an estimated erodibility of $a=187$, which can be compared to the time to breach for Simulation \#1.
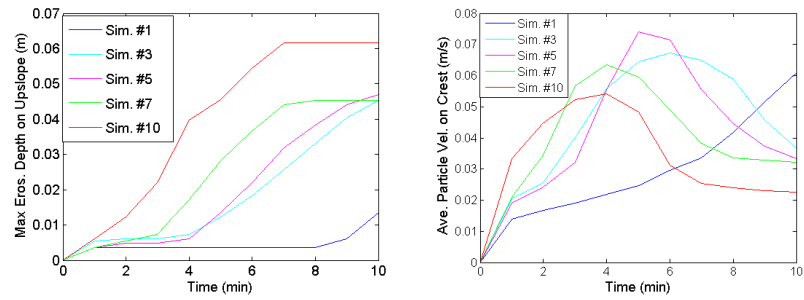

Figure 5: We evaluate two quantitative metrics to define the moment of breach of the levee. The leftmost plot displays the maximum depth of erosion within the zone of the upslope of the levee. The middle plot shows the average velocity of water particles in the zone of the levee crest.
Visually, the progression of the geometric data appears somewhat similar. During the physical experiment, several shallow channels gave way to or joined with a single deep channel that formed along the downslope and slowly eroded back along the crest. Conversely, several computer trials exhibited behavior in which a series of channels formed, though in many cases lesser channel formation did give way to fewer more pronounced channels, with the lesser channels drying up as the experiment progressed. The behavior of the simulated erosion is comparable to that seen in the experiment, especially with regard to the formation and progression of the rills and gullies beginning on the downslope and progressing back across the crest. In both the simulations and the experiment the rill formation starts as the water over-

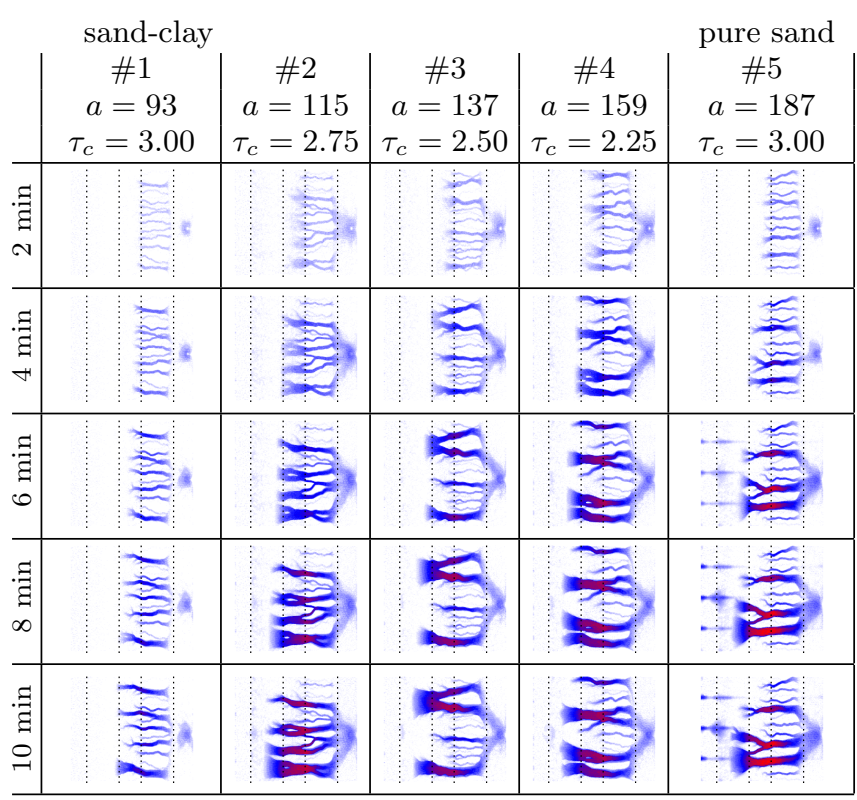

Figure 6: Visualization of the progression of erosion for each of our computer simulations. White indicates no erosion, Erosion ranges from shallow (light blue) to deep (red). Four dashed lines (from left to right) in each image respectively indicate labels $A$, B, C and D in Figure 1. 
Table 1: Erosion parameters and numerical data for the erosion and breaching of our computer simulations.

\begin{tabular}{lccccccc} 
& & & Maximum erosion & Total erosion & \multicolumn{3}{c}{ Time to breach (m:ss) } \\
\cline { 6 - 9 } Simulation & $a$ & $\tau_{c}$ & depth $(\mathrm{m})$ & volume $\left(\mathrm{m}^{3}\right)$ & crest channel & upslope erosion & water velocity \\
\hline \#1 sand-clay mixture & 93 & 3.00 & 0.0450 & 0.0022 & $8: 36$ & $9: 06$ & $7: 15$ \\
\#2 & 115 & 2.75 & 0.0607 & 0.0053 & $3: 13$ & $4: 20$ & $3: 41$ \\
$\# 3$ & 137 & 2.50 & 0.0586 & 0.0047 & $2: 44$ & $4: 08$ & $3: 06$ \\
$\# 4$ & 159 & 2.25 & 0.0577 & 0.0050 & $2: 35$ & $3: 42$ & $2: 24$ \\
\#5 pure sand & 187 & 2.00 & 0.0663 & 0.0091 & $1: 11$ & $1: 06$ & $0: 51$ \\
\hline
\end{tabular}

tops the levee, and continues until the rill has eroded back along the crest. When the rill had reached the upslope, thus breaching the levee, the progression ceased and the water continued to flow along the same channels, slowly cutting away at the edges and bottom and expanding the channels.

The more highly erodible soils in our simulation trials showed significant erosion on the upslope, whereas very little was observed during the experiment. Also, the overall volume of erosion and the depth of erosion of the channels formed during the computer simulation exceeded that of the laboratory experiment, as the channels were carved out faster during the simulation. These discrepancies can be attributed to a number of factors not taken into account by our simulation, such as soil moisture content of the soil, soil porosity, and the presence of a clay or wood levee core, which will have a substantial impact on the erodibility of the soil and the behavior of the water in the system. Also missing from the simulation was deposition, which has a clear impact on the behavior of the water once it reaches the bottom of the downslope, and may or may not affect the erosion along the downslope.

\section{FUTURE WORK}

To improve the accuracy of results, we will extend our simulation engine to include sediment transport and deposition and soil permeability. We will also implement a more physically-accurate model of crumbling overhangs and slumping. To enhance simulation efficiency, we will further optimize our implementation and improve the parallelization strategy to use a super computer or GPUs, allowing us to work with larger datasets at higher resolutions. Furthermore, we will conduct additional physical experiments and acquire higher-resolution digital scans to facilitate precise geometric comparison.

\section{ACKNOWLEDGMENTS}

This research was supported by NSF grant CMMI-0835762.

\section{REFERENCES}

[1] J. L. Briaud, H.-C. Chen, A. V. Govindasamy, and R. Storesund. Levee Erosion by Overtopping in New Orleans during the Katrina Hurricane. Journal of Geotechnical and Geoenvironmental Engineering, 134(618), 2008.

[2] Z. Chen, C. S. Stuetzle, B. M. Cutler, J. A. Gross, W. R. Franklin, and T. F. Zimmie. Analyses, simulations and physical modeling validation of levee and embankment erosion. In To appear in Geo Frontiers 2011, Dallas, Texas, US, 2011. To appear.
[3] N. Foster and D. Metaxas. Realistic animation of liquids. Graphical Models and Image Processing, (58):471-483, 1996.

[4] D. L. Fred. Dambrk: The nws dam-break flood forcasting model. National Weather Service, Office of Hydrology, Silver Spring, Md, 1984.

[5] R. A. Gingold and J. J. Monaghan. Smoothed particle hydrodynamics: Theory and application to no-spherical stars. Mon. Not. R. Astr. Soc., (181):375-389, 1977.

[6] J. A. Gross, C. S. Stuetzle, Z. Chen, B. Cutler, W. R. Franklin, and T. Zimmie. Simulating levee erosion with physical modeling validation. In The 5th International Conference on Scour and Erosion (ICSE 2010), San Fransisco, CA, November 2010. (to appear).

[7] G. J. Hanson, K. R. Cook, and S. L. Hunt. Physical modeling of overtopping erosion and breach formation of cohesive embankments. Transactions of the ASAE, 48(5):1783-1794, 2005.

[8] P. Kristof, B. Benes, J. Krivanek, and O. St'ava. Hydraulic erosion using smoothed particle hydrodynamics. Computer Graphics Forum, 28(2):219-228, 2009.

[9] L. B. Lucy. A numerical approach to the testing of the fission hypothesis. Astronomical Journal, (82):1013-1024, 1977.

[10] C. S. Stuetzle, Z. Chen, B. Cutler, W. R. Franklin, J. Gross, K. Perez, and T. Zimmie. Computer simulations and physical modelling of erosion. In $7 t h$ International Conference on Physical Modelling in Geotechnics (ICPMG 2010), Zurich, June 2010. (to appear).

[11] G. Valette, S. Prevost, L. Lucas, and J. Leonard. SoDA project: A simulation of soil surface degradation by rainfall. Computers \&s Graphics, 30(4):494-506, August 2006.

[12] P. Wang and R. Kahawita. Modeling the hydraulics and erosion process in breach formation due to overtopping. In Sedimentation and Sediment Transport, Proceedings, pages 211-220, 2003.

[13] P. Wang, R. Kahawita, A. Moktari, T. M. Phat, and T. T.Quach. Modeling breach formation in embankments due to overtopping. Transactions of the International Congress on Large Dams, 22(3):377-396, 2006.

[14] T. F. Zimmie. Geo-environmental research using centrifuges. In The Encyclopedia of Environmental Control Technology, volume 9, pages 335-366. Gulf Publishing Co., 1995. 